\title{
Meiosis in autopolyploid Crepis capillaris. III. Comparison of triploids and tetraploids; evidence for non independence of autonomous pairing sites
}

\author{
G. H. JONES \\ School of Biological Sciences, University of Birmingham, Birmingham B15 2TT, U.K.
}

\begin{abstract}
A comparison of meiotic pairing patterns in autopolyploids of Crepis capillaris reveals that the chromosomes of autotriploids form 60 per cent more pairing partner switches (PPSs) than autotetraploids. This difference is highly significant and affects all three chromosomes of Crepis capillaris to about the same extent. However, from theoretical considerations, the chromosomes of autotriploids and autotetraploids are expected to form equal numbers of PPSs provided that they have the same numbers of autonomous pairing sites (APSs) which are acting independently or showing the same degree of dependence and there are no pairing preference differences. Differences in numbers of APSs or in pairing preferences are thought to be unlikely and therefore it is considered that the difference in PPS frequencies reflects some form of interference between APSs affecting the choice of pairing partners but to different degrees in triploids and tetraploids. Such interdependence is fully consistent with the original concept of APSs as 'regions with the capacity to initiate pairing partner exchange in an autopolyploid, regardless of the likelihood of such exchange'.
\end{abstract}

Keywords: autopolyploid, chromosome pairing, Crepis capillaris, meiosis, pairing partner switch.

\section{Introduction}

Valuable information on the organization of meiotic chromosome pairing has been gained by challenging the meiotic process with numerically or structurally modified chromosome sets. In autopolyploids and polysomics, meiotic pairing is generally restricted to pairwise associations but the chromosomes are able to engage different pairing partners at different regions, thus leading to pairing partner switches (PPSs) and multivalents at prophase I (reviewed by Sybenga, 1975; Gillies, 1989). Many cytologists from Newton \& Darlington (1929) onwards have recognized that the special situation in autopolyploids may give unique insights into the organization of pairing, especially as regards the numbers of pairing sites (Klingstedt, 1937; Sved, 1966; Jackson \& Casey, 1982). Further consideration of the situation in autopolyploids has resulted in the concept of the autonomous pairing site (APS) as one which is capable of initiating pairing between any two of three or more homologous chromosomes and therefore of generating a PPS but without specifying the probability that this will occur (Callow \& Gladwell, 1984).

According to this concept an APS is constitutionally capable of acting independently but does not necessarily do so. Two sources of non independent pairing behaviour are recognized.

1 Pairing preferences may exist among the set of homologues so that chromosomes are more likely to pair with particular partners than with others. The effect of pairing preferences will be to reduce the frequencies of PPSs and, in some cases, of multivalents. Such pairing preferences are most likely to occur in allopolyploids but they have been implicated also in autopolyploids (Santos et al., 1983), their extent depending presumably on the levels of genic and molecular heterozygosity in the diploid progenitors of the autopolyploids.

2 The choice of pairing partners at an APS may be influenced by the pairing partner choices at adjacent APSs, even in the absence of pairing preferences. If pairs or groups of APSs tend to act in concert as regards pairing partner choices, the effect will be to 
reduce the frequencies of PPSs. This can be regarded as 'negative pairing interference' in the sense that the choice of pairing partners at one APS makes it more likely that the same chromosomes will pair at adjacent, neighbouring APSs. Positive pairing interference can also be envisaged by which the choice of pairing partners at one APS makes it less likely that the same chromosomes will pair at adjacent APSs.

Non independence of APSs is suggested by some indications from the literature that autotriploids have higher PPS frequencies and more multivalents than autotetraploids. Hamey et al. (1988) found that Crepis rubra has more trivalents in autotriploids than it has quadrivalents in autotetraploids. This information was obtained from high chiasma frequency sets of metaphase I cells and was interpreted as evidence for more PPSs at prophase I in the triploid (Hamey et al., 1988). Similarly, autotriploid Allium sphaerocephalon shows many more PPSs in surface-spread prophase I nuclei than does autotetraploid Allium vineale (Loidl \& Jones, 1986; Loidl, 1986) which is also indicative of a similar trend despite involving an inter specific comparison. In contrast to these observations, theoretical considerations (see below) lead us to expect that corresponding autotriploids and autotetraploids should form the same numbers of APSs provided that pairing organization is similar in terms of APS numbers, that these APSs are acting independently of one another and that pairing preferences are absent or at least do not differ between the ploidy levels.

Although there is an increasing body of data on primary prophase I pairing behaviour in autopolyploids based on SC surface-spreading, until recently it has not been possible to compare directly primary pairing behaviour in autotriploids and autotetraploids of the same plant species. A limited comparison has been possible in the silkworm Bombyx mori females where both autotriploids and autotetraploids have been analysed at prophase I by three-dimensional reconstruction, but this situation is atypical because of the progressive and complete elimination of multivalents during prophase I which is generally regarded as resulting from or reflecting the achiasmate nature of female meiosis in this species (Rasmussen, 1977; Rasmussen \& Holm, 1979).

Extensive analyses of prophase I pairing behaviour have recently been completed in autotriploid and autotetraploid Crepis capillaris based on SC surfacespreading (Vincent \& Jones, 1993; Jones \& Vincent, 1994). These studies provide an ideal basis for direct comparisons of PPS frequencies in autotriploids and autotetraploids of the same species, with the added advantage that each individual chromosome of the Crepis capillaris genome can be unambiguously identi- fied and comparisons made of specific chromosomes at the different ploidy levels.

\section{Materials and methods}

The triploids and tetraploids included in this study originated spontaneously and independently among the progeny of plants recently derived from natural populations of Crepis capillaris occurring on the campus of the University of Birmingham (Vincent \& Jones, 1993; Jones \& Vincent, 1994).

Prophase I nuclei were prepared for electron microscopical examination using a surface-spreading technique developed for plant pollen mother cells (Albini \& Jones, 1984) and later modified for Crepis capillaris by Jones et al. (1989). Suitably spread and silver stained nuclei were transferred to EM grids and examined in a Philips EM301 or a JEOL EX electron microscope. SC configurations were analysed from enlarged photographic prints to determine accurately the numbers of PPSs in trivalents and quadrivalents.

\section{Theoretical considerations}

Two APSs per trisome or tetrasome are required to generate a single PPS in a triploid or tetraploid. When only one APS is present, synapsis proceeds continuously from that point to all other regions involving the same pair or pairs of chromosomes. However, the existence of two or even more than two APSs does not guarantee the production of a PPS as it depends on which chromosomes become paired at each APS. Consider the simple case of an autotriploid with three homologous chromosomes (1,2 and 3 ) and having two APSs ( $a$ and $b$ ) located for convenience at each end of the chromosomes, as shown below.

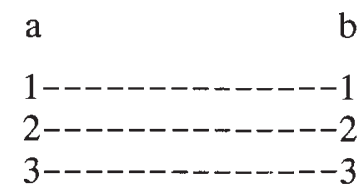

$\begin{array}{cc}\text { PPS } & \begin{array}{c}\text { Prophase I } \\ \text { configuratio }\end{array} \\ 0 & \text { II and I } \\ 1 & \text { III } \\ 1 & \text { III }\end{array}$

$\begin{array}{cccc}a_{1}-a_{2} & b_{1}-b_{2} & 0 & \text { II and I } \\ a_{1}-a_{2} & b_{1}-b_{3} & 1 & \text { III } \\ a_{1}-a_{2} & b_{2}-b_{3} & 1 & \text { III }\end{array}$

If $a_{1}$ and $a_{2}$ pair at site $a$ there are three possibilities at site $b$, two of which result in a PPS and production of a trivalent configuration. Obviously the same applies when pairing at site a involves other combinations $\left(a_{1}-a_{3} ; a_{2}-a_{3}\right)$, giving a total of nine different pairing combinations at $a$ and $b$ of which $2 / 3$ (66.6 per cent) result in a PPS (Sybenga, 1975). 
In an autotetraploid with four homologous chromosomes $(1,2,3,4)$ and the same two APSs ( $a$ and $b$ ), if $a_{1}$ and $a_{2}$ pair at site $a$ then $a_{3}$ and $a_{4}$ are obliged to pair as the other potential partners are occupied, as shown below.

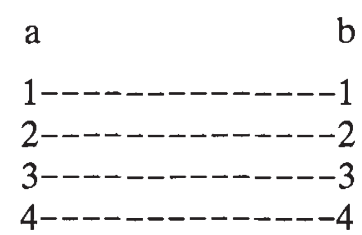

Prophase I

PPS configuration

$\begin{array}{llll}\mathrm{a}_{1}-\mathrm{a}_{2}\left(\mathrm{a}_{3}-\mathrm{a}_{4}\right) & \mathrm{b}_{1}-\mathrm{b}_{2}\left(\mathrm{~b}_{3}-\mathrm{b}_{4}\right) & 0 & \text { 2IIs } \\ \mathrm{a}_{1}-\mathrm{a}_{2}\left(\mathrm{a}_{3}-\mathrm{a}_{4}\right) & \mathrm{b}_{1}-\mathrm{b}_{3}\left(\mathrm{~b}_{2}-\mathrm{b}_{4}\right) & 1 & \text { IV } \\ \mathrm{a}_{1}-\mathrm{a}_{2}\left(\mathrm{a}_{3}-\mathrm{a}_{4}\right) & \mathrm{b}_{1}-\mathrm{b}_{4}\left(\mathrm{~b}_{2}-\mathrm{b}_{3}\right) & 1 & \text { IV }\end{array}$

In this situation there are again three optional pairing combinations at site $b$, two of which give a PPS and a quadrivalent pairing configuration. Again, as in the autotriploid, there are nine possible pairing combinations at $\mathrm{a}$ and $\mathrm{b}$ of which six $(2 / 3$ or 66.6 per cent $)$ give a PPS (Sybenga, 1975).

Thus in the absence of any pairing preferences among homologues and assuming independence of pairing partner choice at two APSs, the probability of a single PPS is $2 / 3$ (66.6 per cent) for both autotriploids and autotetraploids (Jackson \& Casey, 1982; Sybenga, 1975). An additional APS will, on average, generate $2 / 3$ PPS irrespective of whether the trisome or tetrasome already contains one PPS. Consequently the relationship of mean PPS frequency to the numbers of APSs, in both autotriploids and autotetraploids, can be expressed as:

PPS $=\frac{2}{3}($ APS -1$)$

(Loidl \& Jones, 1986; Loidl, 1986) and this relationship should hold over any number of APSs in both triploids and tetraploids provided that APSs are pairing independently and that there are no pairing preferences among the homologues.

\section{Results and discussion}

Detailed qualitative observations and quantitative analyses of meiosis in autotriploid and autotetraploid Crepis capillaris have been previously published (Vincent \& Jones, 1993; Jones \& Vincent, 1994). In this paper attention is focused on a comparison of the mean frequencies of PPSs in these triploids and tetraploids and the relevant data are summarized in Table 1 and Fig. 1. The well-differentiated karyotype of Crepis capillaris allows each individual chromosome to be
Table 1 Mean PPS frequencies for the A, D and C chromosomes separately in autotriploid and autotetraploid Crepis capillaris

\begin{tabular}{ccccc}
\hline & & $\mathrm{A}$ & $\mathrm{D}$ & $\mathrm{C}$ \\
\hline $3 \mathrm{x}$ & $\bar{x}$ & 4.56 & 3.95 & 3.29 \\
& $\mathrm{SD}$ & 1.82 & 2.34 & 2.67 \\
& $n$ & 16 & 19 & 21 \\
$4 \mathrm{x}$ & $\bar{x}$ & 3.04 & 2.59 & 1.64 \\
& $\mathrm{SD}$ & 2.01 & 1.68 & 1.68 \\
& $n$ & 25 & 22 & 25 \\
\hline
\end{tabular}

SD, standard deviation; $n$, no. of observations; $\bar{x}$, mean.

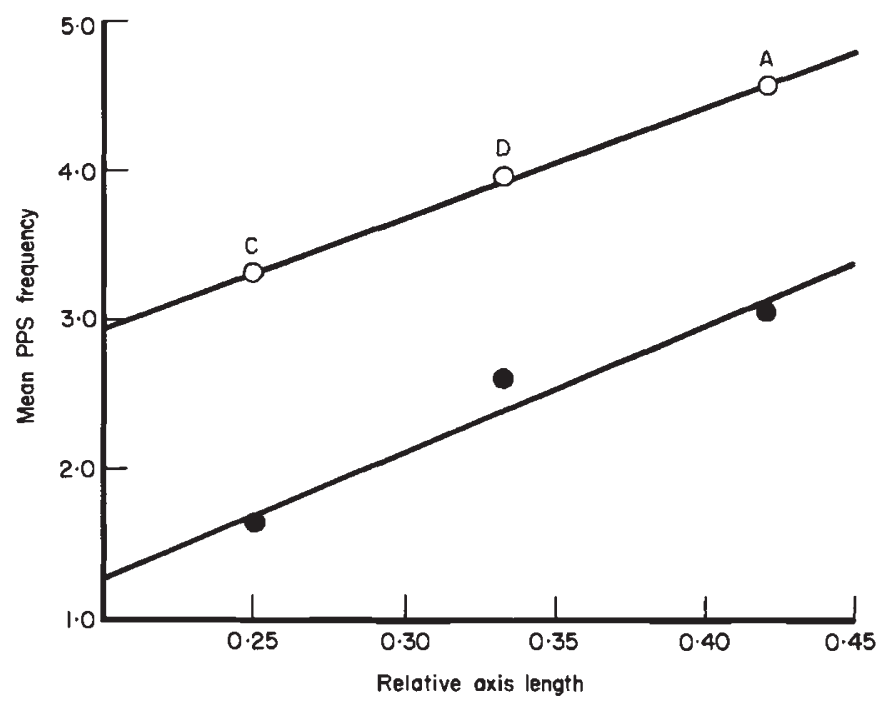

Fig. 1 Mean PPS frequencies plotted against relative axis lengths for the $\mathrm{A}, \mathrm{D}$ and $\mathrm{C}$ chromosomes in autotriploid (open circles) and autotetraploid (solid circles) Crepis capillaris. Lines of best fit, calculated from the regression equations, are included.

separately and unambiguously identified in SC surface spreads (Jones \& de Azkue, 1993) so that the analysis of PPSs can be extended to include a comparison of PPS frequencies at different ploidy levels in different chromosomes. It is evident from Fig. 1 that PPS frequency increases more or less linearly with chromosome size (prophase I axis length), at least over the size range found in the normal Crepis capillaris karyotype. An analysis of variance (Table 2) confirms that the A, $\mathrm{D}$ and $\mathrm{C}$ chromosomes differ significantly for mean PPS frequency over both triploids and tetraploids and furthermore that this includes a highly significant linear regression component. However, the mean frequency of PPSs is consistently higher in the triploid than in the 
Table 2 Analysis of variance of PPS numbers in autotriploid and autotetraploid Crepis capillaris

\begin{tabular}{lrccc}
\hline Item & d.f. & MS & VR & $P$ \\
\hline 1. Chromosomes & 2 & 0.9037 & 4.39 & $<0.05$ \\
a Regression & 1 & 1.7598 & 8.53 & $<0.01$ \\
b Remainder & 1 & 0.0476 & $<1$ & \\
2. Ploidy (3x vs 4x) & 1 & 3.4202 & 16.59 & $<0.001$ \\
3. Ploidy $\times$ chromosomes & 2 & 0.0106 & $<1$ & \\
4. Error & 122 & 0.2062 & & \\
\hline
\end{tabular}

tetraploid nuclei in all three chromosomes. The analysis confirms that this difference between ploidy levels is significant. In addition, the "chromosomes $\times$ ploidy" interaction item is nonsignificant thus showing that the $\mathrm{A}, \mathrm{D}$ and $\mathrm{C}$ chromosomes respond similarly in terms of PPS frequency to the difference in ploidy level.

The main point of interest to emerge from this analysis is that the theoretical expectation of equal PPS frequencies in autotriploids and autotetraploids is not realized in Crepis capillaris. The higher frequency of PPSs in triploids confirms the trend previously noted in Crepis rubra (Hamey et al., 1988) and in an interspecific Allium comparison (Loidl \& Jones, 1986; Loidl, 1986).

Three different factors can be identified which could explain differences in PPS frequency between triploids and tetraploids, or in any other situation, as follows: (1) different numbers of APSs, (2) differences in preferential pairing, and (3) differences in APS interdependence. Considering the first two of these, the observed difference in PPS frequencies might be due to fewer APSs in tetraploids compared with triploids or to differences in the extent of strength of preferential pairing, such that tetraploids exhibit more or stronger pairing preferences. These explanations are considered unlikely because there are no a priori reasons to expect such differences between autotriploids and autotetraploids. The molecular nature of APSs is presently unknown but however they are regarded, either as fixed sites perhaps representing specialized DNA sequences with a role in homology searching, or as unfixed more or less random sites in the genome, there are no grounds for expecting their numbers to differ in a systematic manner between autotriploids and autotetraploids. Similarly, differential pairing affinity is just as likely to occur in autotriploids as in autotetraploids and autotetraploids are therefore not inherently more likely to exhibit preferential pairing. It cannot be ruled out that chance genotypic differences affecting APS number or variety in these particular triploids and tetraploids account for the difference in pairing behaviour. However, the origins of these polyploids from the same natural population material make such differences relatively improbable. Furthermore, it seems very unlikely that such effects, even if they existed, would be equally and evenly distributed over all three chromosomes of the Crepis capillaris genomes in the manner required by the PPS data.

The third possible explanation for the difference and the one considered most likely in view of the arguments presented above (by a process of elimination) is that, contrary to one of the assumptions of the theoretical model presented earlier, APSs are not independent with respect to choice of pairing partner and that the extent or strength of this interdependence differs in a regular and systematic manner between triploids and tetraploids. Since autotetraploids show significantly fewer PPSs compared with autotetraploids, the inference is that tetraploids show negative pairing interference such that the choice of pairing partners at one APS increases the probability that the same chromosomes will pair at adjacent APSs compared with the situation in triploids. This could come about either as a result of a sequential pairing progression whereby the choice of pairing partners at an early site, for example $\mathrm{a}_{1}-\mathrm{a}_{2}$ and $\mathrm{a}_{3}-\mathrm{a}_{4}$ in the model presented earlier, influences the choice of pairing partner at subsequent sites so that, for example, $b_{1}-b_{2}$ and $b_{3}-b_{4}$ are more likely than other pairing combinations. Alternatively, two or more APSs could act simultaneously but co-ordinately with respect to pairing partners, at least in some instances, to give the same result.

Another perspective on this situation is that PPSs may be more frequent in autotriploids because the odd number of chromosomes creates a more competitive pairing situation. In autotriploids three homologous chromosomes compete for pairing partners of which only two can achieve full synapsis at any one APS. The unpaired third chromosome will be available for synapsis elsewhere and indeed will be actively competing for pairing partners at adjacent APSs and this may result in more PPSs than in autotetraploids where all chromosomes are able to pair.

This study confirms previous suggestions from the literature that autotriploids form more PPSs than autotetraploids. One consequence of this is that autotriploids have a higher potential for multivalent formation than do autotetraploids and, indeed, it has been previously noted that autotriploid Crepis rubra forms more trivalents than the autotetraploid forms quadrivalents (Hamey et al., 1988). Since multivalent formation in polyploids is usually negatively correlated with fertility, the inherent tendency of many tetraploids towards low quadrivalent frequencies and high bivalent frequencies clearly has important biological implications and practical applications. 
The inferred interdependence of APSs in polyploid Crepis capillaris clearly illustrates an important and essential characteristic of these hypothetical sites. APSs were originally defined as 'the smallest regions of a chromosome with the capacity to initiate pairing partner exchange ( $=$ PPS) in an autopolyploid, regardless of the likelihood of such exchange' (Callow \& Gladwell, 1984; Hamey et al., 1988). This study supports the view that the probability of PPSs arising from the choice of pairing partners is not a fixed property of APSs but can vary depending on the situation, in this case the level of ploidy.

\section{References}

ALBINI, S. M. AND JONES, G. H. 1984. Synaptonemal complex associated centromeres and recombination nodules in plant meiocytes prepared by an improved surface-spreading technique. Exp. Cell Res., 155, 588-592.

CALLOW, R. S. AND GLADWELL, I. 1984. A general treatment of chromosome synapsis in even-numbered polyploids. $J$. Theor. Biol., 106, 455-494.

GILLIES, C. B. 1989. Chromosome pairing and fertility in polyploids. In: Gillies, C. B. (ed.) Fertility and Chromosome Pairing: Recent Studies in Plants and Animals, pp. 137-176. CRC Press, Boca Raton.

HAMEY, Y., ABBERTON, M. T., WALLACE, A. J. AND CALLOW, R. S. 1988. Pairing autonomy and chromosome size. In: Brandham, P. E. (ed.) Kew Chromosome Conference III, pp. 241-251. Her Majesty's Stationery Office, London.

JACKSON, R. C. AND CASEY, J. 1982. Cytogenetic analyses of autopolyploids: models for triploids to octoploids. Am. J. Bot., 69, 487-501.

JONES, G. H. AND DE AZKUE, D. 1993. Synaptonemal complex karyotyping: an appraisal based on a study of Crepis capillaris. Chromosome Res., 1, 197-203.
JONES, G. H. AND VINCENT, J. E. 1994. Meiosis in autopolyploid Crepis capillaris. II. Autotetraploids. Genome (in press).

JONES, G. H., WHITEHORN, J. A. F. AND ALBINI, S. M. 1989. Ultrastructure of meiotic pairing in $\mathrm{B}$ chromosomes of Crepis capillaris. I. One-B and two-B pollen mother cells. Genome, 32, 611-621.

KLINGSTEDT, H. 1937. On some tetraploid spermatocytes in Chrysochraon dispar. Memor. Soc. Fauna Flora Fenn., 12, 194-209.

LOIDL, J. 1986. Synaptonemal complex spreading in Allium. II. Tetraploid A. vineale. Can. J. Genet. Cytol., 28, 754-761.

LOIDL, J. AND JONES, G. H. 1986. Synaptonemal complex spreading in Allium. I. Triploid A. sphaerocephalon. Chromosoma, 93, 420-428.

NEWTON, W. C. F. AND DARLington, C. D. 1929. Meiosis in polyploids. I. Triploid and pentaploid tulips. J. Genet., 21, $1-15$.

RASMUSSEN, S. W. 1977. Chromosome pairing in triploid females of Bombyx mori analysed by three-dimensional reconstruction of synaptonemal complexes. Carlsberg Res. Commun., 42, 163-197.

RASMUSSEN, S. W. AND HOLM, P. B. 1979. Chromosome pairing in autotetraploid Bombyx females: mechanisms for exclusive bivalent formation. Carlsberg Res. Commun., 44, $101-125$

Santos, J. L., OREllana, J. AND Giraldez, R. 1983. Pairing competition between identical and homologous chromosomes in rye and grasshoppers. Genetics, 104, 677-684.

SVED, J. A. 1966. Telomere attachment of chromosomes. Some genetical and cytological consequences. Genetics, 53, 747-756.

SYBENGA, J. 1975. Meiotic Configurations. Springer, Berlin.

VINCENT, J. E. AND JONES, G. H. 1993. Meiosis in autopolyploid Crepis capillaris. I. Triploids and trisomics; implications for models of chromosome pairing. Chromosoma, 102, $195-206$ 